\title{
REA_12 - Development and standardization of a new method for Virus-Like Particle quantification by digital PCR
}

Pedro Henrique Cardoso ${ }^{1 *}$; Elaine Motta Costa ${ }^{1}$; Marisa de Oliveira Ribeiro ${ }^{1}$; Daniele Ramos Rocha ${ }^{1}$; Elisabete Ferreira de Andrade ${ }^{1}$; Sthefanie da Silva Ribeiro ${ }^{1}$; Marcela Fontana do Carmo Machado Maurell $^{1}$; Daniela Tupy de Godoy ${ }^{1}$; Rodrigo de Moraes Brindeiro ${ }^{2}$; Patrícia Alvarez Baptista ${ }^{1}$.

${ }^{1}$ Fiocruz/Bio-Manguinhos;

${ }^{2}$ UFRJ - Universidade Federal do Rio de Janeiro.

Introduction: The NAT PLUS HIV/HCV/HBV/MAL molecular Kit (Bio-Manguinhos) is used to complement the serological screening in Hemotherapy Services, through the detection of the nucleic acid of hemotransmitted pathogens HIV, HCV, HBV, and Malaria, aiming to increase transfusion safety. Performing an assay with high sensitivity and specificity, this kit has an internal control (IC) to validate the results of each reaction. Protected by a patent (No. PI 0600715-5), the IC is a biosecure Virus-like Particle (VLP), derived from HIV and modified by directed mutagenesis techniques to delete its ability to replicate. During the VLP production, it is essential to know the VLP concentration in each step of the process for quality control. This project has selected the digital PCR (dPCR) to quantify the VLP produced, since it has the advantage of performing absolute quantifications without standards, being mathematically accurate, and easy to operate.

Objective: The objective of this study is to develop and standardize a protocol for VLP quantification by dPCR.

Methodology: 10-fold serial dilutions, ranging from 100x to $1.000 .000 \mathrm{x}$, of a VLP sample recuperated of the production process were done. The NAT Plus platform (JANUS/CHEMAGIC 360, Perkin Elmer ${ }^{\mathbb{R}}$ ) was used to isolate the VLP RNA. After the cDNA synthesis by High-Capacity® cDNA Reverse Transcription Kit, these samples were tested by Quantstudio ${ }^{\text {TM }}$ 3D Digital PCR System. Comparative assays using a range of different primers and TaqMan ${ }^{\circledR}$ FAM-MGB probe concentrations for $15 \mu \mathrm{L}$ final reaction volume were done. The automatic chip loader step distributes the PCR mixture and sample into 20.000 parallel nanoscale reactions. A range of $50^{\circ} \mathrm{C}$ to $60^{\circ} \mathrm{C}$ annealing temperature was made to achieve optimal digital PCR conditions. Poisson Plus statistic model was applied for determining copies/reaction.

Results: The dilution S2 (1000x) was the optimal one for the dPCR quality and quantification parameters analysis. It was at $6,1 \times 10^{5}$ copies $/ \mu \mathrm{L}$ and $2.1 \%$ for percent deviation accuracy (assuming lower and upper $95 \%$ confidence bound) using $54^{\circ} \mathrm{C}$ for annealing temperature, $0,55 \mu \mathrm{M}$ for primers and $0,15 \mu \mathrm{M}$ for FAM probe. The other dilutions points are out of the dPCR precise quantification dynamic range.

Conclusion: It is known that the detection range of $\mathrm{dPCR}$ is stricter compared to real-time RT-PCR quantification assays using standard sample. However, quantification biases can occur, reducing results confidence. These preliminary results indicate the potential of dPCR for VLP absolute quantification tests. More assays are being carried out to improve the accuracy and sensitivity of the dPCR reaction.

Keywords: VLP quantification; Digital PCR; Quality control 